\title{
Exigência de Sódio para Frangos de Corte nas Fases de Crescimento (22 a 42 Dias) e Final (43 a 53 Dias) ${ }^{1}$
}

\author{
José Mauricio Sthel de Barros², Paulo Cezar Gomes³, Luiz Fernando Teixeira Albino ${ }^{3}$, Horacio \\ Santiago Rostagno ${ }^{3}$, Leidimara Feregueti Costa ${ }^{4}$
}

\begin{abstract}
RESUMO - Com o objetivo de determinar as exigências de sódio para frangos de corte durante as fases de crescimento e final, foram utilizados, respectivamente, 720 e 600 frangos, machos e fêmeas, nos períodos de 22 a 42 e de 43 a 53 dias de idade, submetidos a uma dieta basal ( 3.100 e $3.210 \mathrm{kcal} \mathrm{EM/kg}$ e 19,6 e 18,6\% de PB) deficiente em sódio (0,017\%), suplementada com NaCl, correspondente aos níveis 0,$077 ; 0,137 ; 0,197 ; 0,257$ e $0,317 \%$ de sódio total. O delineamento utilizado foi o inteiramente casualizado, em arranjo fatorial $5 \times 2$ (níveis x sexo), com quatro repetições de 18 e 15 aves, respectivamente, por unidade experimental. No final de cada fase, foram avaliados ganho de peso (GP), consumo de ração (CR), conversão alimentar (CA), pesos absoluto (PAA) e relativo (PRA) de adrenal, rendimento (RC) e matéria seca de carcaça (MS) e umidade de cama (de 1 a 53 dias), com as exigências estimadas por meio do modelo de regressão polinomial. Em ambos os sexos, os níveis de sódio influenciaram GP, CA e umidade da cama, não ocorrendo efeito significativo sobre os demais parâmetros. Com base nas características de desempenho, as exigências recomendadas de sódio para a fase de crescimento, estimadas pelo modelo de regressão polinomial, respeitando-se a interpretação biológica, foram de $0,197 \%$ para os machos e, ao menos, de $0,317 \%$ para as fêmeas, enquanto para a fase final foram de 0,216 e $0,245 \%$, respectivamente.
\end{abstract}

Palavras-chave: carcaça, exigências nutricionais, frango de corte, sal, sódio, umidade de cama

\section{Sodium Requirement for Broilers in the Growing (from 22 to 42 Days) and Finishing (from 43 to 53 Days) Phases}

\begin{abstract}
Seven hundred and twenty and six hundred chicks, males and females, from 22 to 42 and from 43 to 53 days old, respectively, fed a basal diet ( 3100 and $3210 \mathrm{kcal} \mathrm{ME} / \mathrm{kg}$ and 19.6 and $18.6 \% \mathrm{CP}$ ) deficient in sodium $(0.017 \%)$, supplemented with $\mathrm{NaCl}$, corresponding to the levels of $.077, .137, .197, .257$ and $.317 \%$ of total sodium were used to determine the sodium requirements for broilers during the growing and final phases. A completely randomized design, in a $5 \times 2$ (levels x sex) factorial arrangement, with four replicates of 18 and 15 chicks, respectively, per experimental unit, was used. Weight gain (WG), feed intake (FI), feed:gain ratio (F/G), adrenal absolute (AAW) and relative (ARW) weights, carcass yield (CY) and dry matter in the carcass (DM) and litter moisture (from 1 to 53 days old) were evaluated at the end of each phase, and the requirements were estimated by means of the polynomial regression model. WG, F/G and litter moisture were influenced by the sodium levels in both sexes, and there was no significant effect on the other parameters. Based on the performance characteristics, the sodium requirements recommended for the growing phase, estimated by the polynomial regression model, considering the biological interpretation, were of .197\% for the males and at least of . $317 \%$ for the females, and for the final phase were of .216 e $.245 \%$, respectively.
\end{abstract}

Key Words: carcass, nutritional requirements, broiler, sodium, salt, litter moisture

\section{Introdução}

A indústria avícola tem apresentado, nas últimas décadas, grande desenvolvimento técnico, em decorrência do desenvolvimento genético das linhagens comerciais de frangos de corte, da maior disponibilidade de novos produtos, da melhoria do manejo e do ambiente de criação, incluindo instalações e condições sanitárias. Tardin (1986) relatou que estas alterações são caracterizadas por intenso dinamismo, fazendo com que os produtos oriundos destes avanços tenham suas demandas nutricionais alteradas com o tempo, tornando-se necessária reavaliação das recomendações nutricionais mínimas.

No campo da nutrição, a importância do sódio passou a ser considerada e estudada a partir da

\footnotetext{
${ }^{1}$ Parte da dissertação apresentada pelo primeiro autor à UFV para obtenção do título de "Magister Scientiae". Projeto financiado pela FAPEMIG.

2 Mestre em Zootecnia - Departamento de Zootecnia, UFV - 36571-000 - Viçosa, MG. E.mail: mauricio.sthel@gruposerrana.com.br

3 Professor - Departamento de Zootecnia, UFV - 36571-000 - Viçosa, MG. Bolsista CNPq. E.mail: pcgomes@ufv.br

${ }^{4}$ Estudante de Mestrado do curso de Zootecnia, DZO, UFV - 36571-000 - Viçosa, MG.
} 
observação que animais em estado de carência apresentavam voraz apetite por sal. Essencialidade demonstrada por estudos pioneiros conduzidos por Ringer (1881), citado por Scott et al. (1969), que provou ser indispensável a presença do mineral na constituição dos meios destinados à cultura de tecido in vitro.

Segundo Maynard et al. (1984), a determinação das exigências de minerais, quando comparada com os demais nutrientes orgânicos, é mais difícil de ser obtida, em decorrência de inúmeros fatores: interrelações entre os diversos elementos minerais e suas correlações com as frações orgânicas; formas e quantidades reais dos minerais presentes na dieta; estado de carência dos animais com relação ao mineral pesquisado, influenciando sua absorção; e diferenças genéticas entre animais da mesma espécie.

Andriguetto et al. (1990) mencionaram que é difícil estudar o sódio e o cloro de forma separada, uma vez que sua suplementação é feita simultaneamente, por meio do uso do sal. Retirando-se o sal das dietas, o sódio aparecerá como primeiro limitante, pois seu nível é menor que o de cloro na maioria dos ingredientes utilizados nas rações.

Segundo Scott et al. (1969) e Welch (1984), citado por Rosado (1988), o conteúdo de sódio no organismo animal pode variar entre 0,11 e $0,13 \%$. Parte deste sódio encontra-se no esqueleto, na forma insolúvel, sendo praticamente inerte no organismo, e a maior porcentagem está presente no líquido extracelular, com aproximadamente $93 \%$ do total de cátions do plasma sangüíneo. Por ser o principal cátion presente nos fluidos extracelulares, sua importância na manutenção das funções vitais normais é bastante conhecida. Ele atua essencialmente no equilíbrio ácido básico e de pressão osmótica corporal, na atividade elétrica das células nervosas e do músculo cardíaco, na permeabilidade celular e na absorção dos monossacarídeos e aminoácidos (Guyton, 1985; Rosado, 1988; Andriguetto et al., 1990).

Níveis marginais de sódio nas rações reduzem a absorção de aminoácidos e monossacarídeos pelo trato gastrointestinal, cujo transporte é altamente dependente da bomba de sódio, com piora nas taxas de ganho de peso e de conversão. Entretanto, os organismos são especialmente hábeis na conservação do sódio corporal, alterando sua excreção quando a ingestão é limitada pelo sistema renina-angiotensina, do hormônio antidiurético (ADH) e do mecanismo da sede (Guyton, 1985).
A despeito da importância nutricional do sódio para frangos de corte, poucas são as pesquisas destinadas ao estabelecimento de suas exigências. Este menor interesse, quando comparado a outros macrominerais, se relacionaria principalmente com o baixo custo de suplementação das rações à base de milho e de farelo de soja, uma vez que as fontes suplementares normalmente empregadas (cloreto ou bicarbonato de sódio) apresentam preços relativamente baixos. Nos últimos anos, entretanto, com a intensificação dos problemas sanitários e de ambiência provocados pelo aumento na umidade de cama com as altas densidades de alojamento, os nutricionistas têm sido estimulados a restringir a inclusão de sódio nas rações, como forma de reduzir a ingestão e excreção de água, sem afetar o desenvolvimento das aves, uma vez que a influência de níveis crescentes de sódio e da relação sódio:cloro sobre a umidade de cama é bastante significativa (Hurwitz et al., 1973; Dilworth et al., 1971; Borges et al., 1996). Este efeito não foi observado por Zanardo (1994), que, ao trabalhar com frangos de corte de 1 a 49 dias de idade, alimentados com níveis crescentes de 0,1 a $0,26 \%$ de sódio nas rações, não encontrou diferenças significativas na umidade de cama.

Murakami et al. (1997a) afirmaram que a exigência de sódio não seria mais que $0,15 \%$ da dieta em ambas as fases de crescimento ( 22 a 42 dias) e final (43 a 56 dias de idade), resultado corroborado por Murakami et al. (2000) para a fase de 22 a 42 dias.

Informações dissonantes com relação às exigências nutricionais de sódio nos períodos de 3 a 6 e de 6 a 8 semanas ocorrem entre o NRC (1994), que cita níveis de 0,15 e $0,12 \%$, respectivamente, e Rostagno et al. (1996), que recomendam $0,20 \%$ de sódio para ambas as fases. Desse modo, torna-se evidente a necessidade constante de se estabelecerem as exigências nutricionais mínimas para frangos de corte, principalmente nas fases de crescimento e final, em virtude do pronunciado consumo de ração no período.

Objetivou-se, com este trabalho, determinar a exigência de sódio para frangos de corte nas fases de crescimento ( 22 a 42 ) e final (43 a 53 dias), com base em estimativas de exigência, estabelecidas por meio do modelo de regressão polinomial (linear e quadrática), pelos parâmetros de desempenho e/ou sua interpretação biológica. 


\section{Material e Métodos}

Foram conduzidos dois experimentos (fases de crescimento e final) nas dependências da Seção de Avicultura do Departamento de Zootecnia da Universidade Federal de Viçosa, nos períodos de 26 de setembro a 16 de outubro e de 17 a 26 de outubro de 1997, respectivamente.

Em ambas as fases, utilizaram-se aves da marca comercial Avian Farms, sexadas, criadas em lotes separados por sexo, em galpão convencional de piso cimentado, coberto com maravalha, contíguo as unidades experimentais. Neste período pré-experimental ou de adaptação (de 1 a 21 dias para o experimento I e de 1 a 42 dias para o experimento II), as aves foram mantidas sob condições tradicionais de manejo, conforme descritas por Gomes et al. (1996), com fornecimento de rações comerciais nas fases inicial e de crescimento.

No experimento I ( 22 a 42 dias), 720 frangos (360 machos e 360 fêmeas) com peso médio inicial respectivo de 718 e $675 \mathrm{~g}$ foram alojados em 40 boxes. Para o experimento II, de 43 a 53 dias de idade, foram utilizados 600 frangos (300 machos e 300 fêmeas), com peso médio inicial de 2.189 e 1.944 g, respectivamente, alocados também em 40 boxes.

Em ambos os experimentos, as aves foram alojadas de forma aleatória, em boxes $\left(2 \mathrm{~m}^{2}\right)$ cobertos com maravalhas, situados em galpão convencional de criação, com 24 horas de luz contínua (natural + artificial). Contudo, uma vez que a montagem dos experimentos era feita seqüencialmente, sem que houvesse a possibilidade de troca do material de cama, a distribuição dos tratamentos nestas fases seguiu o modelo adotado anteriormente na fase de 1 a 21 dias, de forma que todas as unidades experimentais repetissem o mesmo tratamento e sexo nos três experimentos e, assim, a umidade de cama obtida ao término da fase final correspondesse ao período de total 1 a 56 dias de idade.

Os teores de sódio contido na água fornecida às aves durante o período experimental foram monitorados utilizando-se amostras semanais (quatro) e quantitativamente determinados pelo método do espectofotômetro de chama, variando entre 4 e 4,5 ppm, níveis considerados normais segundo Macari (1996). A temperatura e umidade relativa do ar no interior do galpão foram diariamente monitoradas nas duas fases experimentais (Tabela 1).
O delineamento adotado foi o inteiramente casualizado, em arranjo fatorial $5 \times 2$ (cinco níveis de sódio $\mathrm{x}$ dois sexos) e quatro repetições de 18 e 15 aves por unidade experimental para as fases de crescimento (exp. I) e final (exp. II), respectivamente.

As rações experimentais basais foram formuladas à base de milho e farelo de soja (Tabela 2) segundo as recomendações de Rostagno et al. (1996), de modo suprir às exigências nutricionais mínimas de cada fase, exceto para o nutriente em estudo. $\mathrm{Na}$ metade da ração basal de cada fase experimental, o inerte foi parcialmente substituído por $0,770 \%$ de cloreto de sódio (com $39 \%$ de $\mathrm{Na}$ ), correspondendo ao tratamento com $0,317 \%$ de $\mathrm{Na}$ $\left(\mathrm{T}_{5}\right)$ e, em ambas as fases, os demais tratamentos foram obtidos pela diluição desta dieta com a ração basal correspondente, conforme se segue: $\mathrm{T}_{4}=$ com $0,257 \%$ de $\mathrm{Na}\left(80 \%\right.$ de $\mathrm{T}_{5}+20 \%$ da basal $) ; \mathrm{T}_{3}=$ com $0,197 \%$ de $\mathrm{Na}\left(60 \%\right.$ de $\mathrm{T}_{5}+40 \%$ da basal); $\mathrm{T}_{2}=$ com $0,137 \%$ de $\mathrm{Na}\left(40 \%\right.$ de $\mathrm{T}_{5}+$ $60 \%$ da basal); e $\mathrm{T}_{1}=$ com $0,077 \%$ de $\mathrm{Na}(20 \%$ de $\mathrm{T}_{5}+80 \%$ da basal).

No final de cada fase (42ํ e 53ำ dia), foram avaliados o ganho de peso, o consumo de ração e a conversão alimentar das aves. Para avaliação do rendimento e da matéria seca das carcaças e dos tamanhos absoluto e relativo das glândulas adrenais, foram abatidas três aves por unidade experimental, totalizando 24 aves por tratamento (12 machos e 12 fêmeas), na média de peso do boxe, sacrificadas após oito horas de jejum.

Todas as adrenais foram retiradas, identificadas e resfriadas em solução fisiológica para posterior avaliação de peso. Na pesagem (em balança analítica de 0,0001 g de precisão), as glândulas adrenais foram secas em papel-filtro e limpas com bisturi, retirando possíveis tecidos não-pertencentes ao órgão. As carcaças, após resfriamento, foram pesadas e processadas conjuntamente (por unidade experimental) em moinho tipo Cutter, durante 20 minutos. Amostras foram retiradas e congeladas para posterior análise de umidade, segundo o método descrito por Silva (1990).

Com o término da fase final e a retirada dos animais, todo o material de cama foi homogeneizado dentro de cada boxe e amostrado, para determinação da umidade, conforme método descrito por Silva (1990). Os resultados obtidos corresponderam, ao período total de criação (1 a 53 dias), uma vez que os experimentos foram realizados de forma consecutiva 
(sem que houvesse a troca do material de cama entre as fases) e que, cada tratamento (nível de sódio e sexo) foi montado de forma a se repetir nas fases pesquisadas dentro da mesma unidade experimental. Para determinação do rendimento de carcaça, foi considerado o peso da carcaça eviscerada resfriada, incluindo cabeça, pés e gordura abdominal, em relação ao peso vivo individual, obtido no pré-abate.

As análises estatísticas dos parâmetros foram processadas utilizando-se o SAEG - Sistema de Análises Estatísticas e Genéticas (UFV, 1997) e, as exigências, estimadas pelo modelo de regressão polinomial, considerando o coeficiente de determinação $\left(\mathrm{r}^{2}\right)$ e a sua interpretação biológica. As análises de variância foram realizadas segundo o modelo matemático:

$$
\mathrm{Y}_{\mathrm{ijk}}=\mu+\mathrm{T}_{\mathrm{j}}+\mathrm{S}_{\mathrm{i}}+\mathrm{TS}_{\mathrm{ij}}+\varepsilon_{\mathrm{ijk}}
$$

em que $\mathrm{Y}_{\mathrm{ijk}}=$ produção observada na unidade experimental $\mathrm{k}$, que recebeu o nível de sódio j e o sexo $\mathrm{i}$; $\mu=$ média geral; $\mathrm{T}_{\mathrm{j}}=$ efeito do $\mathrm{j}^{\mathrm{j} \text { éssimo }}$ nível de sódio, sendo $\mathrm{j}=0,077 ; 0,137 ; 0,197 ; 0,257$ e $0,317 \%$; $\mathrm{S}_{\mathrm{i}}=$ efeito do $\mathrm{i}^{\text {-éssimo }}$ sexo, sendo $\mathrm{i}=$ macho e fêmea; $\mathrm{TS}_{\mathrm{ij}}=$ efeito da interação nível de sódio j e do sexo i; e $\varepsilon_{\mathrm{ijk}}=$ erro aleatório associado a cada observação, sendo o erro NID $\left(0, \mathrm{~S}^{2}\right)$.

\section{Resultados e Discussão}

Os efeitos dos níveis de sódio sobre o desempenho de frangos de corte, machos e fêmeas, nos períodos de 22 a 42 dias e de 43 a 53 dias de idade, são apresentados nas Tabelas 3 e 4, respectivamente.
Em concordância com os dados obtidos por Dewar \& Whitehead (1973), houve efeito significativo dos níveis de sódio, no período de crescimento (22 a 42 dias), sobre o ganho de peso e a conversão alimentar das aves, com efeito quadrático para os frangos machos e linear para as fêmeas, em ambas as características. No entanto, Zanardo (1994), Borges et al. (1996) e Murakami et al. (1997a) não observaram melhora significativa no desempenho, de 22 a 42 dias, com crescentes níveis de sódio nas rações.

Para o período final (43 a 53 dias), o ganho de peso e a conversão alimentar das aves de ambos os sexos foram influenciados significativamente pelos níveis de sódio, corroborando os resultados obtidos por Dilworth et al. (1971). Houve efeito quadrático do sódio para ambas as características, independentemente do sexo. Entretanto, Murakami et al. (1997a) não observaram melhoras significativas no desempenho de frangos de corte machos, de 42 a 56 dias de idade, quando os níveis de sódio das rações variaram de 0,15 a $0,30 \%$.

Segundo Walicka et al. (1979), aves carentes em $\mathrm{NaCl}$ elevam sua taxa metabólica basal na tentativa de controlar a homeostasia corporal, com perda de eficiência alimentar e retardo no crescimento (baixo ganho de peso), o que explicaria a queda de desempenho obtido pelo estudo, em ambas as fases pesquisadas, de machos e fêmeas, nos níveis inferiores de sódio. Independentemente do sexo, nas fases de crescimento e final, não houve efeito significativo dos níveis de sódio pesquisados sobre o consumo de ração, o que está de acordo com os resultados obtidos por Borges et al. (1996) e Murakami et al. (1997a).

Tabela 1 - Temperatura e umidade relativa do ar no interior do galpão, durante as fases de crescimento (22 a 42 dias) e final ( 43 a 53 dias)

Table 1 - Temperature and air relative moisture in the floor pens, during the growing and final phases

\begin{tabular}{|c|c|c|c|c|c|}
\hline \multirow[t]{2}{*}{$\begin{array}{l}\text { Temperatura do ar }\left({ }^{\circ} \mathrm{C}\right) \\
\text { Air temperature }\end{array}$} & \multicolumn{5}{|c|}{$\begin{array}{c}\text { Idade das aves (dias) } \\
\text { Chicks age (days) }\end{array}$} \\
\hline & $22-28$ & $29-35$ & $36-42$ & $43-49$ & $50-53$ \\
\hline $\begin{array}{l}\text { Média das máximas } \\
\text { Maximum mean }\end{array}$ & $29,1 \pm 2,4$ & $27,8 \pm 2,5$ & $31,1 \pm 2,8$ & $24,8 \pm 2,9$ & $28,6 \pm 2,3$ \\
\hline $\begin{array}{l}\text { Média das mínimas } \\
\text { Minimum mean }\end{array}$ & $22,0 \pm 2,1$ & $18,4 \pm 2,1$ & $20,3 \pm 2,8$ & $19,4 \pm 1,6$ & $20,3 \pm 2,1$ \\
\hline $\begin{array}{l}\text { Máxima absoluta } \\
\text { Absolute maximum }\end{array}$ & 31 & 31 & 34 & 30 & 30 \\
\hline $\begin{array}{l}\text { Mínima absoluta } \\
\text { Absolute minimum }\end{array}$ & 19 & 15 & 16 & 18 & 18 \\
\hline $\begin{array}{l}\text { Umidade relativa }(\%) \\
\text { Relative moisture }\end{array}$ & $82,3 \pm 6,8$ & $88,6 \pm 4,7$ & $89,5 \pm 2,2$ & $94,3 \pm 1,9$ & $93,3 \pm 2,9$ \\
\hline
\end{tabular}

R. Bras. Zootec., v.33, n.6, p.1721-1733, 2004 (Supl. 1) 
Tabela 2 - Composição percentual da ração basal para frangos de corte nas fases de crescimento ( 22 a 42 dias) e final ( 43 a 53 dias)

Table 2 - Percentage composition of the basal diet fed to broilers in the growing (22 to 42 days old) and final (43 to 53 days old) phases

\begin{tabular}{|c|c|c|}
\hline $\begin{array}{l}\text { Ingrediente } \\
\text { Ingredient }\end{array}$ & $\begin{array}{l}\text { Crescimento }(\%) \\
\text { Growing }\end{array}$ & $\begin{array}{l}\text { Final }(\%) \\
\text { Final }\end{array}$ \\
\hline Milho - 8,5\% PB & & \\
\hline $\begin{array}{l}\text { Corn }-8.5 \% \text { CP } \\
\text { Farelo de soja }-45,5 \% \text { PB }\end{array}$ & 57,87 & 60,18 \\
\hline $\begin{array}{l}\text { Soybean meal - } 45.5 \% C P \\
\text { Óleo }\end{array}$ & 33,22 & 30,62 \\
\hline $\begin{array}{l}\text { Oil } \\
\text { Calcário }\end{array}$ & 4,05 & 5,12 \\
\hline $\begin{array}{l}\text { Limestone } \\
\text { Fosfato bicálcico }\end{array}$ & 1,33 & 1,37 \\
\hline $\begin{array}{l}\text { Dicalcium phosphate } \\
\text { Suplemento mineral }^{1}\end{array}$ & 1,55 & 1,32 \\
\hline $\begin{array}{l}\text { Mineral premix } \\
\text { Suplemento vitamínico }{ }^{2}\end{array}$ & 0,05 & 0,05 \\
\hline $\begin{array}{l}\text { Vitamin premix } \\
\text { Antioxidante }(\mathrm{BHT})\end{array}$ & 0,10 & 0,10 \\
\hline $\begin{array}{l}\text { Antioxidant } \\
\text { Bacitracina de zinco }\end{array}$ & 0,01 & 0,01 \\
\hline $\begin{array}{l}\text { Zinc bacitracin } \\
\text { Cloreto de colina }(60 \%)\end{array}$ & 0,02 & 0,02 \\
\hline $\begin{array}{l}\text { Choline chlorine } \\
\text { Anticoccidiano }^{3}\end{array}$ & 0,06 & 0,04 \\
\hline $\begin{array}{l}\text { Anticoccidial } \\
\text { DL-metionina (99\%) }\end{array}$ & 0,06 & 0,06 \\
\hline $\begin{array}{l}\text { DL-methionine } \\
\text { L-lisina. } \mathrm{HCl}(78,4 \%)\end{array}$ & 0,13 & 0,11 \\
\hline $\begin{array}{l}\text { L-lysine } \\
\text { Inerte (caulim) }\end{array}$ & 0,05 & 0,00 \\
\hline Inert & 1,50 & 1,00 \\
\hline TOTAL & 100,00 & 100,00 \\
\hline
\end{tabular}

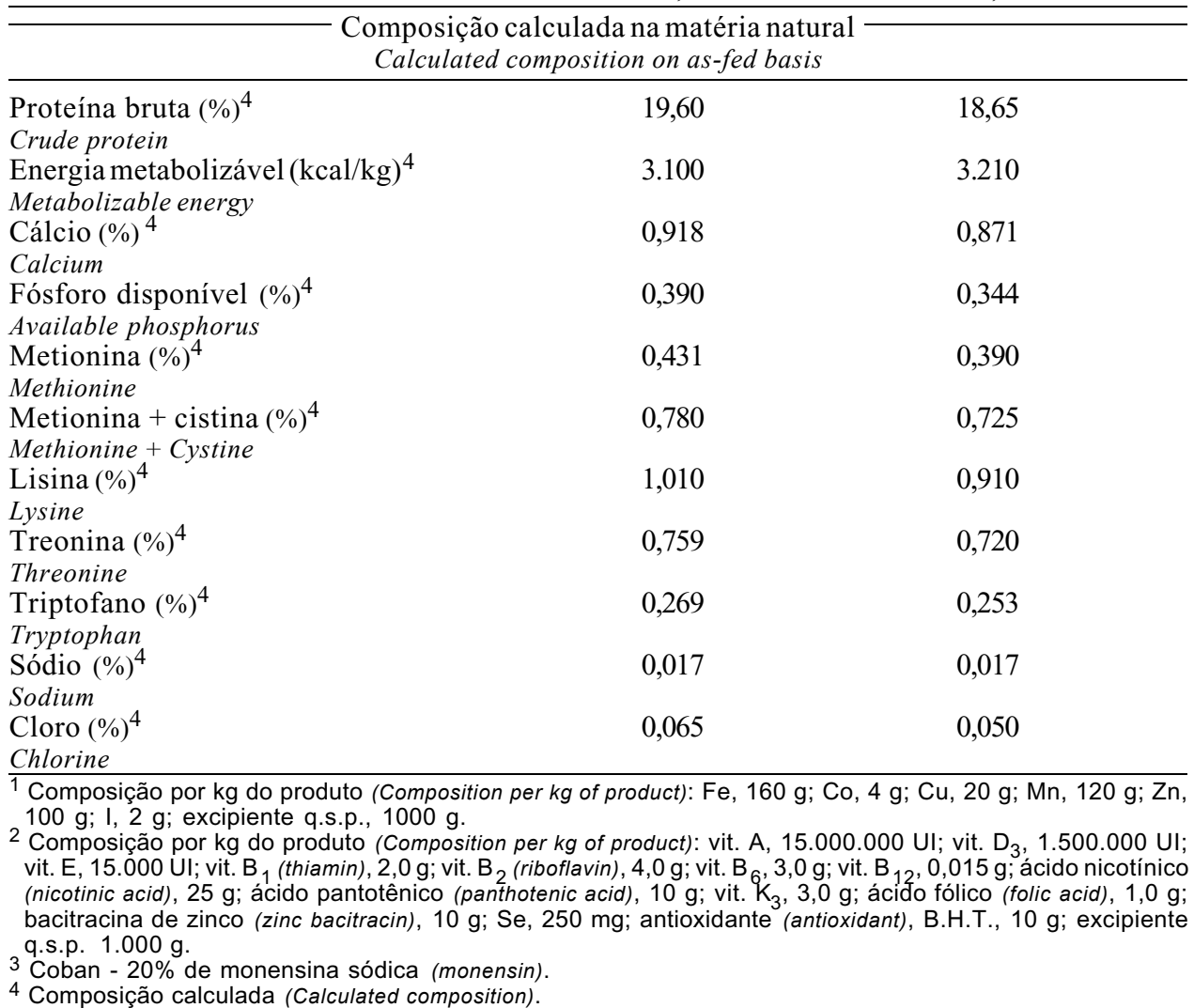

\section{R. Bras. Zootec., v.33, n.6, p.1721-1733, 2004 (Supl. 1)}


Portanto, melhor ganho de peso não seria uma resposta a possíveis incrementos no consumo de ração, conforme proposto por Penz Jr. (1998). Segundo o autor, aumento de consumo de ração estaria relacionado à maior ingestão de água pelas aves alimentadas com níveis mais elevados de sódio. Murakami et al. (1997b) observaram, entretanto, que o consumo de ração não era significativamente influenciado pelos teores de sódio, mas sim pelos níveis de cloro das rações.

Em ambas as fases estudadas, machos e fêmeas diferiram em todas as características de desempenho avaliadas, tendo os machos apresentado maior ganho de peso, maior consumo de ração e melhor conversão alimentar que as fêmeas, o que era esperado. Estes resultados diferem dos observados por Borges et al. (1996) e Zanardo (1994), que não encontraram diferenças significativas para ganho de peso de machos e fêmeas no período de 22 a 42 dias.

$\mathrm{O}$ efeito quadrático dos níveis de sódio sobre o desempenho de frangos machos de 22 a 42 dias e de machos e fêmeas de 43 a 53 dias de idade sinaliza que os níveis superiores de sódio, empregados no estudo, comprometem de alguma forma o seu desempenho.
Algumas hipóteses sugerem que esta queda seria de forma direta: pela toxidez do mineral nos níveis superiores ou por aumento do gasto de energia pela bomba de Na-K para o controle da homeostase corporal. Indiretamente, pela piora na qualidade de cama (úmida), o que a torna mais propensa a proliferação de agentes causadores de doenças. No período de 22 a 42 dias de idade, o maior nível de sódio nas rações das fêmeas foi acompanhado por aumento linear no ganho de peso e na conversão alimentar, sugerindo maior exigência por parte das fêmeas.

Os efeitos dos níveis de sódio sobre o rendimento e matéria seca de carcaça, tamanhos relativo e absoluto das adrenais de frangos de corte, machos e fêmeas, no $42^{\circ}$ e $53^{\circ}$ dia de idade, são apresentados nas Tabelas 5 e 6 , respectivamente.

Barlow et al. (1948) verificaram que altos níveis de sal na ração tendiam a equivocar o ganho de peso e o peso vivo das aves pelo aumento na retenção de água pelos tecidos corporais. Entretanto, os resultados obtidos não demonstram tal fato, já que, em ambas as fases estudadas, independentemente do sexo, os níveis de sódio não influenciaram a matéria

Tabela 3 - Efeito dos diferentes níveis de sódio sobre ganho de peso (GP), consumo de ração (CR) e conversão alimentar (CA) de frangos de corte (22 a 42 dias de idade $)^{1}$

Table 3 - Effect of different sodium levels on the weight gain (WG), feed intake (FI) and feed:gain ratio (F/G) of broiler chicks (from 22 to 42 days of age)

\begin{tabular}{|c|c|c|c|c|}
\hline $\begin{array}{l}\text { Sexo } \\
\text { Sex }\end{array}$ & $\begin{array}{l}\text { Sódio }(\%) \\
\text { Sodium }\end{array}$ & $\begin{array}{c}\text { Ganho de peso }(\mathrm{g}) \\
\text { Weight gain }\end{array}$ & $\begin{array}{l}\text { Consumo de ração }(\mathrm{g}) \\
\text { Feed intake }\end{array}$ & $\begin{array}{c}\text { Conversão alimentar } \\
\text { Feed:gain ratio }\end{array}$ \\
\hline \multirow[t]{2}{*}{$\begin{array}{l}\text { Macho } \\
\text { Male }\end{array}$} & $\begin{array}{l}0,077 \\
0,137 \\
0,197 \\
0,257 \\
0,317\end{array}$ & $\begin{array}{l}1.471,78 \\
1.532,39 \\
1.640,73 \\
1.583,25 \\
1.601,20\end{array}$ & $\begin{array}{l}2.973,88 \\
2.965,51 \\
3.099,64 \\
3.019,55 \\
3.026,87\end{array}$ & $\begin{array}{l}2,021 \\
1,936 \\
1,889 \\
1,908 \\
1,891\end{array}$ \\
\hline & Média (Mean) & $1.565,87$ & $3.017,09$ & 1,929 \\
\hline \multirow[t]{2}{*}{$\begin{array}{l}\text { Fêmea } \\
\text { Female }\end{array}$} & $\begin{array}{l}0,077 \\
0,137 \\
0,197 \\
0,257 \\
0,317\end{array}$ & $\begin{array}{l}1.268,11 \\
1.298,85 \\
1.307,12 \\
1.321,81 \\
1.341,95\end{array}$ & $\begin{array}{l}2.627,38 \\
2.629,44 \\
2.644,78 \\
2.662,56 \\
2.653,69\end{array}$ & $\begin{array}{l}2,072 \\
2,024 \\
2,023 \\
2,014 \\
1,977\end{array}$ \\
\hline & Média (Mean) & $1.307,56$ & $2.643,57$ & 2,022 \\
\hline $\begin{array}{l}\text { Níveis de sódio }{ }^{2} \\
\text { Sodium levels }\end{array}$ & $\begin{array}{l}\text { Macho } \\
\text { Male } \\
\text { Fêmea } \\
\text { Female }\end{array}$ & $\begin{array}{l}\mathrm{Q} * * * \\
\mathrm{~L} * *\end{array}$ & $n s$ & $\begin{array}{l}\mathrm{Q} * * \\
\mathrm{~L} * * *\end{array}$ \\
\hline \multicolumn{2}{|c|}{$\begin{array}{l}\text { Sexo } \\
\text { Sex }\end{array}$} & $* * *$ & $* * *$ & $* * *$ \\
\hline \multicolumn{2}{|c|}{ CV $(\%)$} & 2,47 & 1,96 & 1,64 \\
\hline
\end{tabular}

$1 * * *(P \leq 0,001),{ }^{* *}(P \leq 0,01)$ e "ns" $(P>0,05)$, pelo teste $F . ~ " n s "=$ não-significativo.

$1 * * *(P \leq .001),{ }^{* *}(P \leq .01)$ and "ns" $(P>.05)$, by $F$ test. "ns"= not significant.

$Q=$ Efeito quadrático. $L=$ Efeito linear.

$Q=$ Quadratic effect. $L=$ Linear effect.

R. Bras. Zootec., v.33, n.6, p.1721-1733, 2004 (Supl. 1) 
seca das carcaças evisceradas, resultados que corroboram os obtidos por Dilworth et al. (1971), Dewar \& Whitehead (1973) e Zanardo (1994), que também não observaram influência dos níveis de sódio no teor de água corporal em idades diversas. Nas fases de crescimento e final, os frangos machos apresentaram menor porcentagem de matéria seca nas carcaças que as fêmeas.

Barlow et al. (1948), ao descreverem o acúmulo de líquidos corporais em rações com altos níveis de sal, não citam em quais tecidos prevalecia esta retenção. Portanto, foi determinado o rendimento de carcaça das aves, de forma que, caso a retenção ocorresse nas vísceras, menor rendimento seria observado. Resultado semelhante foi obtido por Murakami et al. (1997a), que não observaram alteração de rendimento em diferentes níveis de sódio para frangos abatidos aos 56 dia, em ambos os períodos e sexos. Esses autores verificaram que o nível de sódio das rações não alterou o rendimento de carcaça das aves, comprovando que o maior ganho de peso observado não foi provocado por maior quantidade de água corporal retida nas vísceras. Se este acúmulo fosse verdadeiro, seria esperada queda no rendimento.

A hipertrofia da zona glomerular do córtex da supra-renal seria uma tentativa de se produzir maior quantidade dos hormônios mineralocorticóides (principalmente aldosterona), responsáveis pelo aumento da capacidade de reabsorção do sódio pelo rins, em baixos níveis do mineral (Guyton, 1985). Entretanto, independentemente da fase estudada e do sexo das aves, os pesos absoluto e relativo das adrenais não foram influenciados significativamente pelos teores de sódio pesquisados, fato não observado por Nott \& Combs (1969), que verificaram efeito significativo do sódio para o peso relativo de adrenal. A falta de resposta aos níveis estudados, a partir da avaliação biométrica, pode estar mais relacionada aos altos coeficientes de variação obtidos para as duas variáveis, com os níveis carenciais, mas não tão severos para as fases pesquisadas, e à maior capacidade de adaptação das aves com o avanço da idade.

Independentemente do sexo, a umidade da cama no período total de criação foi significativamente influenciada pelos níveis de sódio das rações, o que está de acordo com Dilworth et al. (1971), Hurwitz et

Tabela 4 - Efeito dos diferentes níveis de sódio sobre o ganho de peso (GP), o consumo de ração (CR) e a conversão alimentar (CA) de frangos de corte (43 a 53 dias de idade) ${ }^{1}$

Table 4 - Effect of different sodium levels on the weight gain (WG), feed intake (FI) and feed:gain ratio (F/G) of broiler chicks (from 43 to 53 days of age)

\begin{tabular}{|c|c|c|c|c|}
\hline $\begin{array}{l}\text { Sexo } \\
\text { Sex }\end{array}$ & $\begin{array}{l}\text { Sódio (\%) } \\
\text { Sodium }\end{array}$ & $\begin{array}{c}\text { Ganho de peso }(\mathrm{g}) \\
\text { Weight gain }\end{array}$ & $\begin{array}{c}\text { Consumo de ração }(\mathrm{g}) \\
\text { Feed intake }\end{array}$ & $\begin{array}{l}\text { Conversão alimentar } \\
\text { Feed:gain ratio }\end{array}$ \\
\hline \multirow{6}{*}{$\begin{array}{l}\text { Macho } \\
\text { Male }\end{array}$} & 0,077 & 745,83 & 1892,49 & 2,538 \\
\hline & 0,137 & 806,67 & 1926,90 & 2,389 \\
\hline & 0,197 & 807,15 & 1926,09 & 2,386 \\
\hline & 0,257 & 821,66 & 1904,41 & 2,318 \\
\hline & 0,317 & 773,33 & 1894,58 & 2,455 \\
\hline & Média & 790,93 & 1908,89 & 2,417 \\
\hline \multirow{6}{*}{$\begin{array}{l}\text { Fêmea } \\
\text { Female }\end{array}$} & 0,077 & 559,16 & 1607,50 & 2,879 \\
\hline & 0,137 & 605,18 & 1602,34 & 2,648 \\
\hline & 0,197 & 628,30 & 1632,00 & 2,599 \\
\hline & 0,257 & 605,00 & 1612,91 & 2,666 \\
\hline & 0,317 & 620,84 & 1639,58 & 2,641 \\
\hline & Média & 603,70 & 1618,86 & 2,686 \\
\hline \multirow{4}{*}{$\begin{array}{l}\text { Níveis de sódio }{ }^{2} \\
\text { Sodium levels }\end{array}$} & Macho & $\mathrm{Q}^{* * *}$ & $n s$ & $\mathrm{Q} * * *$ \\
\hline & Male & & & \\
\hline & Fêmea & $\mathrm{Q}^{*}$ & $n s$ & $\mathrm{Q}^{* * *}$ \\
\hline & Female & & & \\
\hline \multicolumn{2}{|c|}{ Sexo } & & & \\
\hline \multicolumn{2}{|c|}{ Sex } & $* * *$ & $* * *$ & $* * *$ \\
\hline \multicolumn{2}{|c|}{ CV (\%) } & 3,44 & 1,74 & 2,91 \\
\hline
\end{tabular}

$1 * * *(P \leq 0,001),{ }^{*}(\mathrm{P} \leq 0,05)$ e "ns" $(\mathrm{P}>0,05)$, pelo teste $\mathrm{F}$. "ns"= não-significativo.

$1 * * *(P \leq .001),{ }^{*}(P \leq .05)$ and "ns" $(P>.5)$, by $F$ test. "ns"= not significant.

$Q=$ Efeito quadrático.

$Q=$ Quadratic effect.

R. Bras. Zootec., v.33, n.6, p.1721-1733, 2004 (Supl. 1) 
al. (1973), Borges et al. (1996) e Murakami et al. (1997a). O crescimento linear na umidade (Figura 1), com o aumento dos níveis de sódio estudados, estaria relacionado à maior ingestão de água pelas aves, na tentativa de manter a homeostasia corporal. Pela equação de regressão, obtida para machos e fêmeas, tem-se que, para cada $0,06 \%$ de sódio, ocorrem aumentos de 3,9\% na umidade da cama. Quando se analisam os valores ponto por ponto, observa-se que até o nível de $0,197 \%$ de sódio ocorrem aumentos discretos na porcentagem de umidade da cama, que aumenta em, aproximadamente, $8 \%$ (em números absolutos), quando o teor de sódio sobe para $0,257 \%$, caracterizando o limite em que as aves aumentam o consumo de água como forma de controlar os níveis de sódio das rações.

Estimativas de exigência nutricional em sódio

As exigências em sódio para frangos de corte, machos e fêmeas, de 22 a 42 e de 43 a 53 dias de idade, considerando os dados de desempenho (Tabela 8), foram estimadas a partir de equações ajustadas por modelos de regressão polinomial
(Figuras 2 a 5 ).

$\mathrm{Na}$ fase de 22 a 42 dias de idade, os machos tiveram exigência estimada, respectivamente, em 0,249 e $0,257 \%$, com base no ganho de peso e na conversão

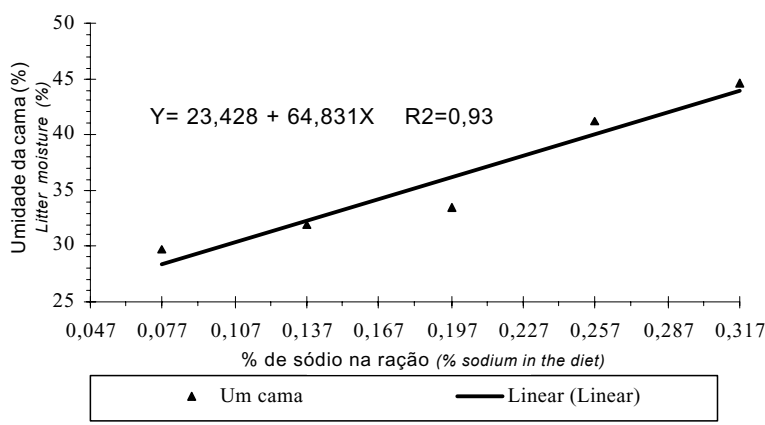

Figura 1 - Efeito dos níveis de sódio da ração sobre a umidade da cama de frangos de corte (1 a 53 dias de idade).

Figure 1 - Effect of dietary sodium levels on the broiler litter moisture (1 to 53 days of age).

Tabela 5 - Efeito dos diferentes níveis de sódio sobre o rendimento (RC) e matéria seca de carcaça (MS), pesos absoluto (PAA) e relativo (PRA) das glândulas adrenais de frangos de corte no $42^{\circ}$ dia de idade ${ }^{1}$

Table 5 - Effect of different sodium levels on the carcass yield (CY), carcass dry matter (CDM), adrenal absolute weight (AAW) and adrenal relative weight (ARW) of broiler chicks at $42^{\text {th }}$ days old

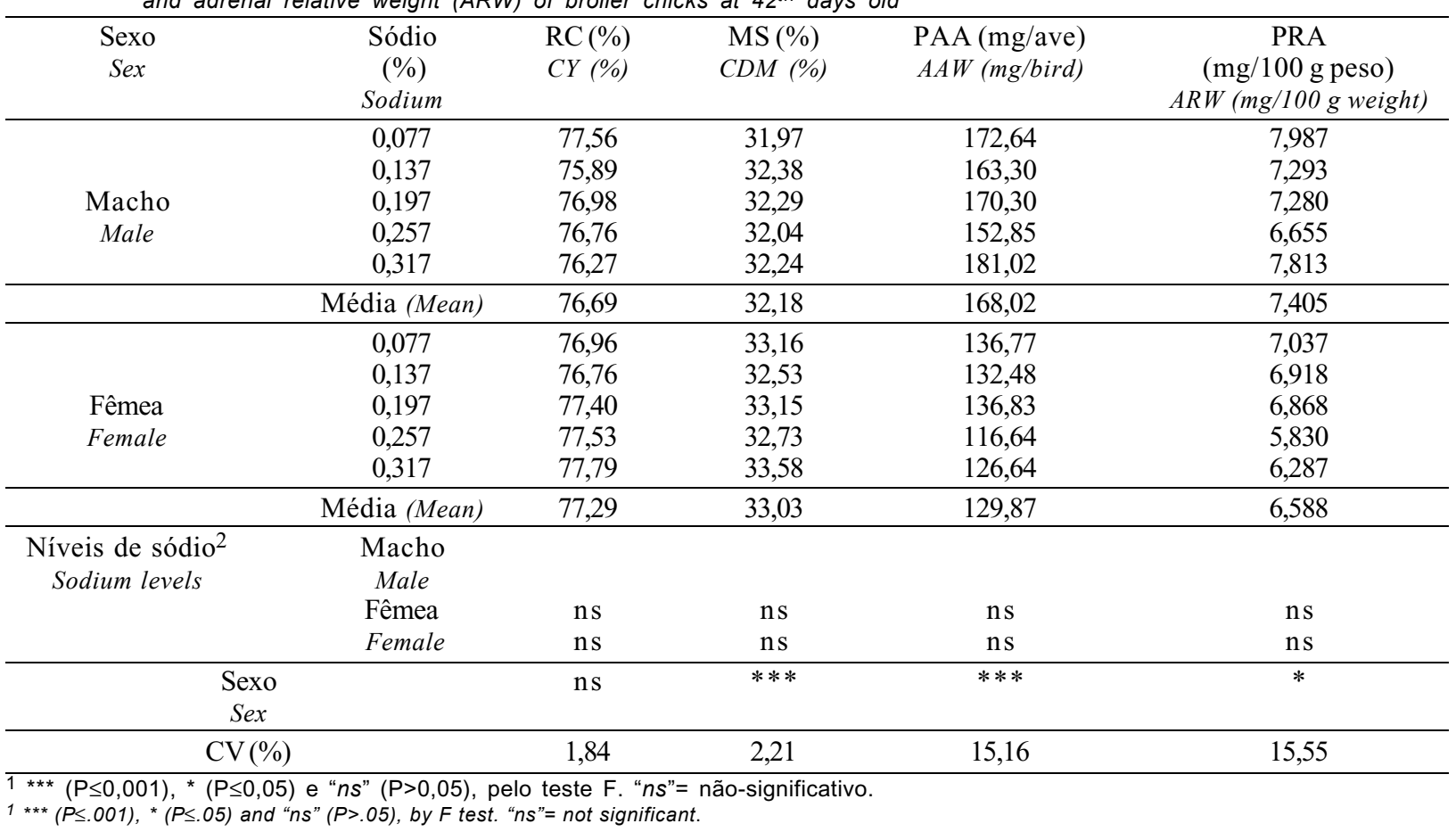

R. Bras. Zootec., v.33, n.6, p.1721-1733, 2004 (Supl. 1) 
Tabela 6 - Efeito dos diferentes níveis de sódio sobre o rendimento (RC) e matéria seca de carcaça (MS), pesos absoluto (PAA) e relativo (PRA) das glândulas adrenais de frangos de corte no 53 o dia de idade ${ }^{1}$

Table 6 - Effect of different sodium levels on the carcass yield (CY), carcass dry matter (CDM), adrenal absolute weight (AAW) and adrenal relative weight $(A R W)$ of broiler chicks at $53^{\text {th }}$ days old

\begin{tabular}{|c|c|c|c|c|c|}
\hline $\begin{array}{l}\text { Sexo } \\
\text { Sex }\end{array}$ & $\begin{array}{c}\begin{array}{c}\text { Sódio } \\
(\%) \\
\text { Sodium }\end{array} \\
\end{array}$ & $\begin{array}{l}\mathrm{RC}(\%) \\
C Y(\%)\end{array}$ & $\begin{array}{l}\text { MS (\%) } \\
C D M(\%)\end{array}$ & $\begin{array}{l}\text { PAA (mg/ave) } \\
A A W(m g / b i r d)\end{array}$ & $\begin{array}{c}\text { PRA } \\
\text { (mg/100 g peso }) \\
A R W(m g / 100 \text { g weight })\end{array}$ \\
\hline & 0,077 & 81,78 & 34,17 & 195,60 & 6,59 \\
\hline $\begin{array}{l}\text { Macho } \\
\text { Male }\end{array}$ & $\begin{array}{l}0,137 \\
0,197 \\
0,257 \\
0,317 \\
\end{array}$ & $\begin{array}{l}81,83 \\
82,15 \\
81,88 \\
80,47 \\
\end{array}$ & $\begin{array}{l}33,78 \\
32,73 \\
32,69 \\
33,03 \\
\end{array}$ & $\begin{array}{l}208,58 \\
201,00 \\
209,35 \\
201,62 \\
\end{array}$ & $\begin{array}{l}6,93 \\
6,68 \\
6,98 \\
6,65 \\
\end{array}$ \\
\hline & Média (Mean) & 81,62 & 33,28 & 203,23 & 6,77 \\
\hline $\begin{array}{l}\text { Fêmea } \\
\text { Female }\end{array}$ & $\begin{array}{l}0,077 \\
0,137 \\
0,197 \\
0,257 \\
0,317 \\
\end{array}$ & $\begin{array}{l}81,79 \\
81,52 \\
81,42 \\
81,70 \\
81,96 \\
\end{array}$ & $\begin{array}{l}33,56 \\
34,04 \\
34,95 \\
35,40 \\
33,70 \\
\end{array}$ & $\begin{array}{l}170,05 \\
152,91 \\
150,51 \\
140,84 \\
154,72 \\
\end{array}$ & $\begin{array}{l}6,79 \\
5,98 \\
5,83 \\
5,53 \\
6,09 \\
\end{array}$ \\
\hline & Média (Mean) & 81,68 & 34,33 & 153,80 & 6,04 \\
\hline $\begin{array}{l}\text { Níveis de sódio }{ }^{2} \\
\text { Sodium levels }\end{array}$ & $\begin{array}{l}\text { Macho } \\
\text { Male } \\
\text { Fêmea } \\
\text { Female }\end{array}$ & $\begin{array}{l}\mathrm{ns} \\
\mathrm{ns}\end{array}$ & $\begin{array}{l}\mathrm{ns} \\
\mathrm{ns}\end{array}$ & $\begin{array}{l}\mathrm{ns} \\
\mathrm{ns}\end{array}$ & $\begin{array}{l}\mathrm{ns} \\
\mathrm{ns}\end{array}$ \\
\hline $\begin{array}{l}\text { Sexo } \\
\text { Sex }\end{array}$ & & $\mathrm{ns}$ & $*$ & $* * *$ & $* *$ \\
\hline CV $(\%)$ & & 1,22 & 3,63 & 11,59 & 12,25 \\
\hline
\end{tabular}

$1 * * *(P \leq 0,001),{ }^{* *}(P \leq 0,01),{ }^{*}(P \leq 0,05)$ e ns $(P>0,05)$, pelo teste $F ;$ ns $=$ não-significativo.

$1 * * *(P \leq .001),{ }^{* *}(P \leq .001),{ }^{*}(P \leq .05)$ and "ns" $(P>.05)$, by $F$ test. "ns"= not significant.

Tabela 7 - Efeito dos diferentes níveis de sódio sobre a umidade da cama de frangos de corte, machos e fêmeas, de 1 a 53 dias de idade ${ }^{1}$

Table 7 - Effect of different sodium levels on the litter moisture of chicks broiler, male and female ( 1 to 53 days old)

\begin{tabular}{|c|c|c|c|}
\hline \multirow{3}{*}{\multicolumn{2}{|c|}{$\begin{array}{l}\text { Níveis de sódio }(\%) \\
\quad \text { Sodium levels }\end{array}$}} & \multicolumn{2}{|c|}{$\begin{array}{l}\text { Sexo } \\
\text { Sex }\end{array}$} \\
\hline & & $\begin{array}{l}\text { Macho } \\
\text { Male }\end{array}$ & $\begin{array}{l}\text { Fêmea } \\
\text { Female }\end{array}$ \\
\hline & & \multicolumn{2}{|c|}{$\begin{array}{l}\text { Umidade da cama }(\%) \\
\text { Litter moisture (\%) }\end{array}$} \\
\hline & 0,077 & 29,33 & 30,24 \\
\hline & 0,137 & 32,31 & 31,53 \\
\hline & 0,197 & 34,30 & 32,65 \\
\hline & 0,257 & 42,25 & 40,23 \\
\hline & 0,317 & 47,02 & 42,13 \\
\hline & Média (Mean) & 37,04 & 35,36 \\
\hline \multicolumn{4}{|c|}{ Níveis de sódio ${ }^{2}$} \\
\hline & Sodium levels & $\mathrm{L} * * *$ & $\mathrm{~L} * * *$ \\
\hline \multicolumn{4}{|c|}{ Sexo } \\
\hline & Sex & \multicolumn{2}{|c|}{ * } \\
\hline & CV $(\%)$ & \multicolumn{2}{|c|}{5,36} \\
\hline P & $\begin{array}{l}* * *(P \leq 0,001) e^{*}(P \leq 0,05) \text { pelo teste } F . \\
* * *(P \leq .001) \text { and }{ }^{*}(P \leq .05) \text { by } F \text { test. }\end{array}$ & & \\
\hline 2 & $\begin{array}{l}L=\text { Efeito Linear. } \\
L=\text { Linear effect. }\end{array}$ & & \\
\hline
\end{tabular}

R. Bras. Zootec., v.33, n.6, p.1721-1733, 2004 (Supl. 1) 
alimentar. Contudo, do ponto de vista biológico, a melhor resposta para ambas as características estão próximas de $0,197 \%$ de sódio total, demostrando que a estimativa obtida por meio do modelo quadrático superestimou o nível ótimo. Para as fêmeas, em virtude do efeito linear observado tanto para ganho quanto conversão, a exigência mínima foi estimada em, pelo menos, $0,317 \%$, ou seja, o maior valor estudado.

A exigência em sódio de 43 a 53 dias de idade, ajustadas por meio do modelo de regressão quadrática, com base no ganho de peso e na conversão, foram, respectivamente, de 0,211 e 216\%, para os machos, e de 0,245 e $0,233 \%$, para as fêmeas.

Em ambas as fases, as fêmeas apresentaram valores de exigência bastante superiores aos estimados para os machos, em decorrência, provavelmente, de fatores como padrão de crescimento tardio da linhagem pesquisada e alto grau de empenamento apresentado pelas fêmeas. Maior empenamento, associado a picos de calor (acima da faixa termoneutra) nos períodos, propicia aumento do nível de estresse das fêmeas. Segundo Smith \& Teeter (1993), melhores desempenhos em frangos estressados pelo calor foram obtidos com a adição de eletrólitos $(\mathrm{KCl}$ e $\mathrm{NaCl})$ na água de bebida. Segundo os autores, o efeito benéfico resulta do maior consumo de água, reduzindo o estresse pelo maior tunover e, possivelmente, por ajustes fisiológicos ainda desconhecidos.

Com base nas respostas de desempenho obtidas em função do nível de sódio, ajustadas por meio do modelo de regressão polinomial, respeitando-se a interpretação biológica, recomenda-se para o período de 22 a 42 dias exigência de sódio de $0,197 \%$, para machos, e de pelo menos $0,317 \%$, para fêmeas. Estes resultados foram superiores aos citados pelo NRC (1994) e por Murakami et al. (1997a) e Murakami et al. (2000), que recomendam 0,15\% de sódio, para ambos os sexos.

Para o período de 43 a 53 dias, a exigência mínima recomendada foi escolhida de forma atender simultaneamente ganho de peso e conversão alimentar, ou seja, o maior valor encontrado entre
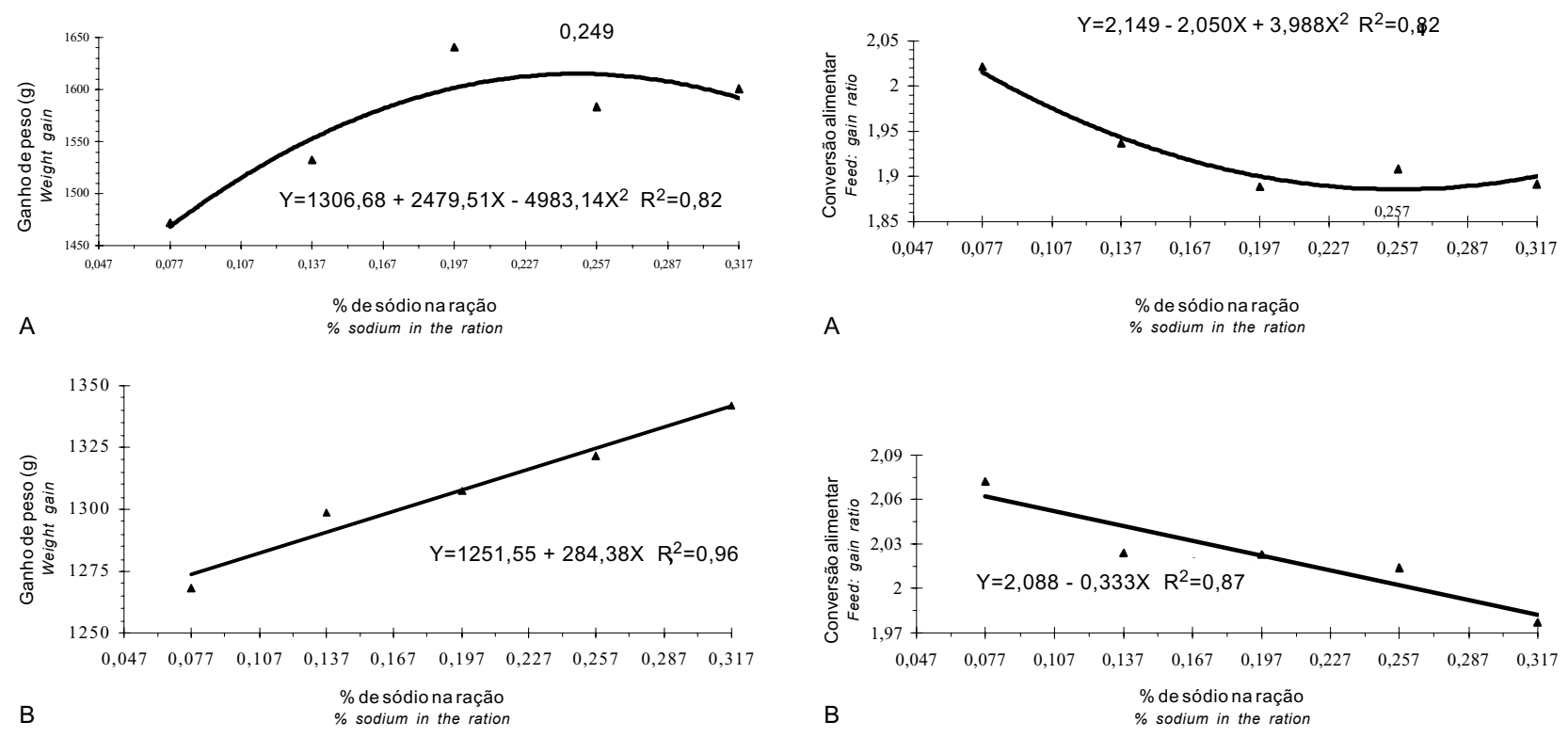

Figura 2 - Efeito dos níveis de sódio da ração sobre o ganho de peso de frangos de corte, machos (A) e fêmeas (B), de 22 a 42 dias de idade.

Figure 2 - Effect of dietary sodium levels on weight gain of male and female chick broilers (22 to 42 days of age).

Figura 3 - Efeito dos níveis de sódio da ração sobre a conversão alimentar de frangos de corte, machos e fêmeas de 22 a 42 dias de idade.

Figure 3 - Effect of dietary sodium levels on feed:gain of male (A) and female (B) chick broilers (22 to 42 days old).

R. Bras. Zootec., v.33, n.6, p.1721-1733, 2004 (Supl. 1) 
Tabela 8 - Estimativa de exigência de sódio para frangos de corte, machos e fêmeas, de 22 a 42 e de 43 a 53 dias de idade, por modelos de regressão ${ }^{1}$

Table 8 - Estimate of sodium requirement for broiler chicks, females and males, from 22 to 42 days and 43 to 53 days old, by the regression models ${ }^{1}$

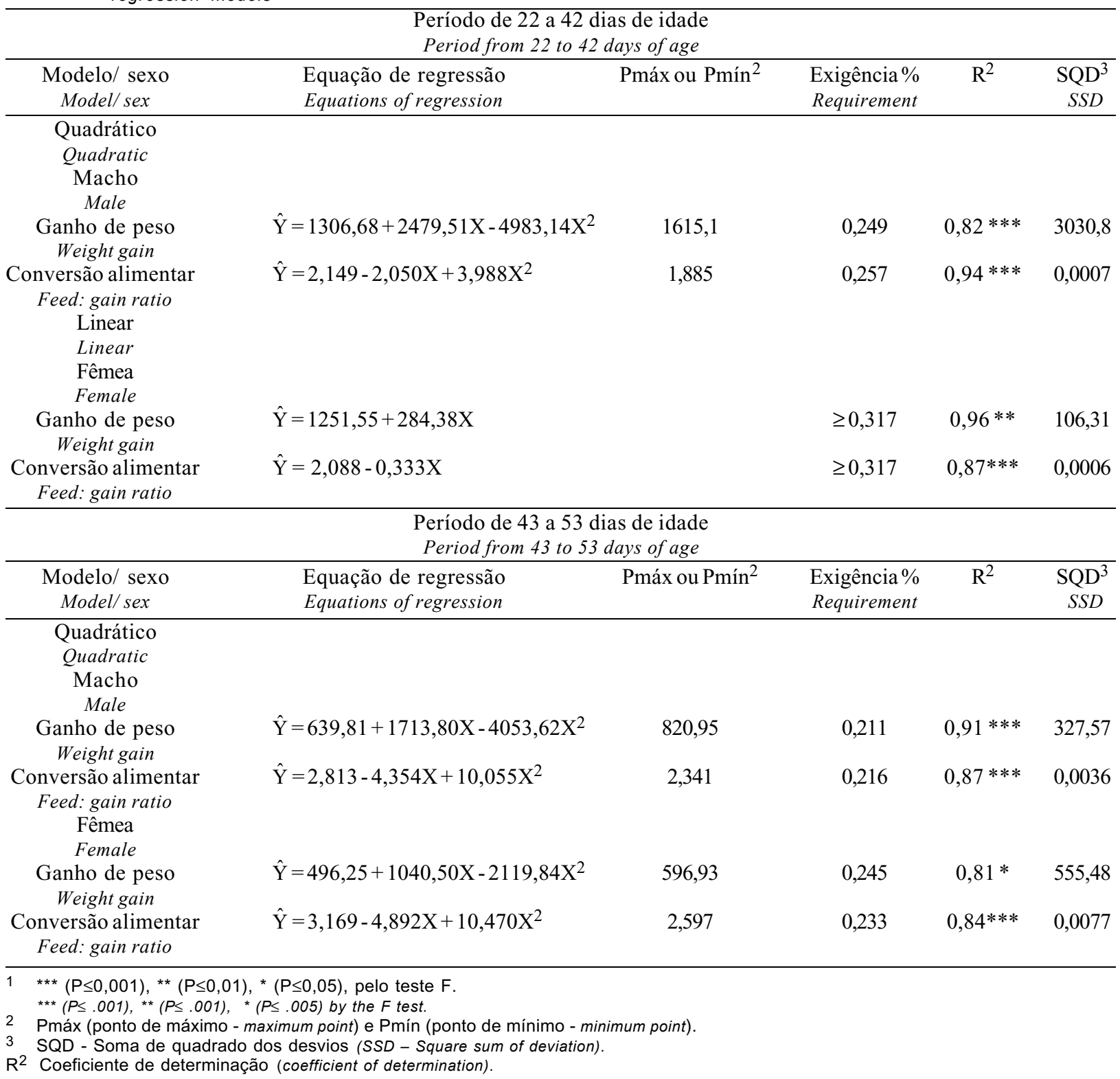

ambos, dentro de cada sexo $(0,216$ e $0,245 \%$, respectivamente, para machos e fêmeas). Estes níveis são superiores ao citado no NRC (1994) como de $0,12 \%$ de sódio para machos e fêmeas. Entretanto, os valores recomendados para frangos machos, tanto na fase de crescimento quanto na fase final, estão próximos dos sugeridos por Rostagno et al. (1996), de 0,20\% de sódio (para ambas as fases), independentemente do sexo.

R. Bras. Zootec., v.33, n.6, p.1721-1733, 2004 (Supl. 1)

\section{Conclusões}

Com base nas características de desempenho obtidas para frangos de corte, de ambos os sexos, respeitando-se a interpretação biológica, recomenda-se, para o período de 22 a 42 dias de idade, exigência nutricional mínima de sódio de $0,197 \%$, para os machos, e de ao menos $0,317 \%$, para as fêmeas. Para o período de 43 a 53 dias de idade, a recomendação é de 0,216 e $0,245 \%$, para machos e fêmeas, respectivamente. 

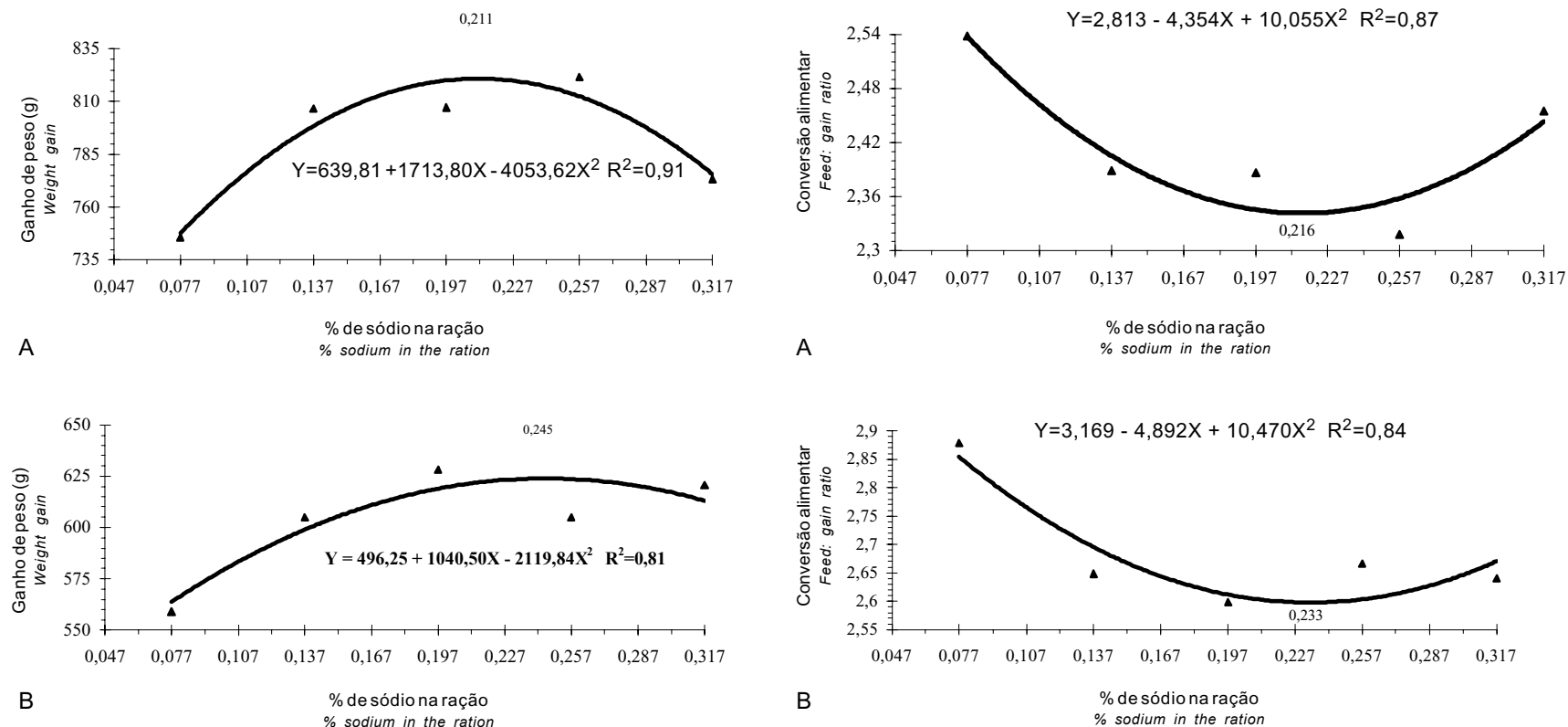

Figura 4 - Efeito dos níveis de sódio da ração sobre o ganho de peso de frangos de corte, machos (A) e fêmeas (B), de 43 a 53 dias de idade.

Figure 4 - Effect of dietary sodium levels on weight gain of male and female broiler chicks (43 to 53 days old).

Figura 5 - Efeito dos níveis de sódio da ração sobre a conversão alimentar de frangos de corte, machos (A) e fêmeas (B), de 43 a 53 dias de idade.

Figure 5 - Effect of dietary sodium levels on feed:gain ration of male and female broiler chicks (43 to 53 days old).

\section{Literatura Citada}

ANDRIGUETTO, J.M.; PERLY, L.; MINARDI, I. et al. Nutricão animal. 4.ed. São Paulo: Nobel, 1990. v.1, 395p.

BARLOW, J.S.; SLINGER, S.J.; ZIMMER, R.P. The reaction of growing chicks to diets varying in sodium chloride content. Poultry Science, v.27, n.5, p.542-552, 1948.

BORGES, S.A.; ARIKI, J.; JERÔNIMO JR., R. et al. Níveis de sal $(\mathrm{NaCl})$ em rações de frangos de corte. In: CONFERÊNCIA APINCO DE CIÊNCIA E TECNOLOGIA AVÍCOLAS, 1996, Curitiba. Anais... Curitiba: FACTA, 1996. p.27 (Anexo).

DEWAR, W.A.; WHITEHEAD, C.C. Sodium supplementation of broiler rations. British Poultry Science, v.14, n.3, p.315-318, 1973

DILWORTH, B.C.; SCHULTZ, C.D.; DAY, E.J. et al. Observations with broilers fed varying dietary sodium chloride levels from 56 to 63 days of age. In: ANNUAL PFIZER RESEARCH CONFERENCE, 19., 1971, Chicago. Proceedings... Chicago: Agricultural division PFIZER INC, 1971. 98p.

GOMES, P.C.; ALBINO, L.F.T.; SILVA, M.A. Criação de frangos de corte. Viçosa, MG: Universidade Federal de Viçosa, 1996. 18p.

GUYTON, A.C. Tratado de fisiologia médica. 8.ed. Rio de Janeiro: Guanabara \& Koogan, 1985. 864p.

HURWITZ, S.; COHEN, I.; BAR, A. et al. Sodium and chloride requirements of the chick: relationship to acid-base balance. Poultry Science, v.52, n.3, p.903-909, 1973.

MACARI, M. Água na avicultura industrial. 1.ed. Jaboticabal: FUNEP, 1996. 128p.

MAYNARD, L.A.; LOOSLY, J.K.; HINTZ, H.F. et al. Nutrição animal. 3.ed. Rio de Janeiro: Freitas Bastos, 1984. 726p.

MURAKAMI, A.E.; SALEH, E.A.; ENGLAND, J.A. et al. Effect of level and source of sodium on performance of male broilers to 56 days. Journal of Applied Poultry Research, v.6, n.1, p.128-136, 1997a.

MURAKAMI, A.E.; WATKINS, S.E.; SALEH, E.A. et al. Estimation of the sodium and chloride requirements for the young broiler chick. Journal of Applied Poultry Research, v.6, n.1, p.155-162, $1997 \mathrm{~b}$.

MURAKAMI, A.E.; RONDON, E.O.O.; SCAPINELLO C. et al. Exigências nutricionais de sódio e cloro para frangos de corte na fase de crescimento ( 22 a 42 dias de idade). In: REUNIÃO ANUAL DA SOCIEDADE BRASILEIRA DE ZOOTECNIA, 37., 2000, Viçosa, MG. Anais... Viçosa, MG: Sociedade Brasileira de Zootecnia, 2000. p.296.

NATIONAL RESEARCH COUNCIL - NRC. Nutrients requirements of poultry. 9.ed. Washington, D.C.: National Academic Press, 1994.

NOTT, H., COMBS, G.F. Sodium requirement of the chick. Poultry Science, v.48, n.2, p.660-665, 1969.

PENZ JR., A.M. Nutrição na primeira semana In: SIMPÓSIO INTERNACIONAL SOBRE MANEJO DE PINTOS DE CORTE NA $1^{\text {a }}$ SEMANA, 1998, Campinas. Anais... Campinas: FACTA, 1998. p.121-139. 
ROSADO, A.M.S. Efeitos de coccidicidas ionofóricos sobre o desempenho e o balanço de eletrólitos em frangos de corte. Viçosa, MG: Universidade Federal de Viçosa, 1988. 75p. Dissertação (Mestrado em Zootecnia) - Universidade Federal de Viçosa, 1988.

ROSTAGNO, H.S.; BARBARINO JR., P.; BARBOSA, W.A Exigências nutricionais das aves determinadas no Brasil. In: SIMPÓSIO INTERNACIONAL SOBRE EXIGÊNCIAS NUTRICIONAIS DE AVES E SUÍNOS, Viçosa, MG, 1996. Anais... Viçosa, MG: Universidade Federal de Viçosa, 1996. p.361-388.

SCOTT, M.L.; NESHEIM, M.S.; YOUNG, R.J. Nutrition of the chicken. New York: M.L. Scott \& Associates, 1969. $511 \mathrm{p}$.

SILVA, D.J. Análise de alimentos: métodos químicos e bromatológicos. Viçosa, MG: Universidade Federal de Viçosa, 1990. 160p

SMITH, M.O.; TEETER, R.G. Carbon dioxide, ammonium chloride, potassium chloride and performance of heat distressed broilers. Journal of Applied Poultry Research, v.2, n.1, p.61-66, 1993.
TARDIN, A.C. Requerimentos nutricionais de matrizes pesadas: programas de alimentação. In: SEMINÁRIO DOS PRODUTORES DE PINTOS DE CORTE, 4., 1986, Campinas. Anais... Campinas: FACTA, 1986. p.29-43.

UNIVERSIDADE FEDERAL DE VIÇOSA - UFV. Sistema de análises estatísticas e genética - SAEG. Viçosa, MG: UFV (Software). 1997.

WALICKA, E.; RYS, R.; KORELESKI, J. et al. Effect of sodium chloride deficiency on basal metabolism in broiler chickens. British Journal Nutrition, v.42, n.3, p.547-552, 1979.

ZANARDO, J.A. Níveis de sódio e agentes anticoccidianos ionóforos e não-ionóforos em rações de frangos de corte. Viçosa, MG: Universidade Federal de Viçosa, 1994. 63p. Dissertação (Mestrado em Zootecnia) - Universidade Federal de Viçosa, 1994.
Recebido em: 22/07/01 Aceito em: 19/08/03 